\title{
Serum levels of neuron-specific ubiquitin carboxyl-terminal esterase-L1 predict brain injury in a canine model of hypothermic circulatory arrest
}

\author{
George J. Arnaoutakis, MD, ${ }^{\mathrm{a}}$ Timothy J. George, MD, ${ }^{\mathrm{a}}$ Kevin K. Wang, PhD, ${ }^{\mathrm{h}}$ Mary Ann Wilson, PhD, ${ }^{\mathrm{b}, \mathrm{c}, \mathrm{g}}$ \\ Jeremiah G. Allen, MD, ${ }^{a}$ Chase W. Robinson, MHS, ${ }^{a}$ Kara A. Haggerty, BS, ${ }^{a}$ Eric S. Weiss, MD, MPH, ${ }^{a}$ \\ Mary E. Blue, PhD, ${ }^{\text {b,c, }}$ Charles C. Talbot, Jr, BA, Juan C. Troncoso, MD, ${ }^{\text {b,e }}$ Michael V. Johnston, MD, ${ }^{\text {b,d,g }}$ and \\ William A. Baumgartner, MD $^{\mathrm{a}}$
}

\begin{abstract}
Objectives: Ubiquitin carboxyl-terminal esterase-L1 (UCHL1) is a protein highly selectively expressed in neurons and has been linked to neurodegenerative disease in humans. We hypothesize that UCHL1 would be an effective serum biomarker for brain injury as tested in canine models of hypothermic circulatory arrest (HCA) and cardiopulmonary bypass (CPB).
\end{abstract}

\begin{abstract}
Methods: Dogs were exposed to CPB $(\mathrm{n}=14), 1$ hour of HCA ( $1 \mathrm{~h}-\mathrm{HCA} ; \mathrm{n}=11)$, or 2 hours of HCA ( $2 \mathrm{~h}-\mathrm{HCA}$; $\mathrm{n}=20$ ). Cerebrospinal fluid and serum were collected at baseline, 8 hours, and 24 hours after treatment. UCHL1 levels were measured using a sandwich enzyme-linked immunosorbent assay. Neurologic function and histopathology were scored at 24 hours, and UCHL1 immunoreactivity was examined at 8 hours.
\end{abstract}

Results: Baseline UCHL1 protein levels in cerebrospinal fluid and serum were similar for all groups. In serum, UCHL1 levels were elevated at 8 hours after treatment for $2 \mathrm{~h}$-HCA subjects compared with baseline values $(P<.01)$ and also compared with CPB dogs at 8 hours $(P<.01)$. A serum UCHL1 level above $3.9 \mathrm{ng} /(\mathrm{mg}$ total protein) at 8 hours had the best discriminatory power for predicting functional disability. In cerebrospinal fluid, UCHL1 was elevated in all groups at 8 hours after treatment compared with baseline $(P<.01)$. However, UCHL1 levels in cerebrospinal fluid remained elevated at 24 hours only in $2 \mathrm{~h}$-HCA subjects $(P<.01)$. Functional and histopathologic scores were closely correlated (Pearson coefficient, $0.66 ; P<.01$ ) and were significantly worse in $2 \mathrm{~h}-\mathrm{HCA}$ animals.

Conclusions: This is the first report associating elevated serum UCHL1 with brain injury. The novel neuronal biomarker UCHL1 is increased in serum 8 hours after severe neurologic insult in $2 \mathrm{~h}$-HCA animals compared with CPB animals. These results support the potential for use in cardiac surgery patients and form the basis for clinical correlation in humans. (J Thorac Cardiovasc Surg 2011;142:902-10)

Supplemental material is available online.

From the Division of Cardiac Surgery, ${ }^{\mathrm{a}}$ Departments of Neurology, ${ }^{\mathrm{b}}$ Neuroscience, ${ }^{\mathrm{c}}$ and Pediatrics, ${ }^{\mathrm{d}}$ Division of Neuropathology, ${ }^{\mathrm{e}}$ Institute for Basic Biomedical Sciences ${ }^{\mathrm{f}}$ at The Johns Hopkins Medical Institutions and the Hugo W. Moser Research Institute at Kennedy-Krieger, ${ }^{\mathrm{g}}$ Baltimore, Md; and Banyan Biomarkers, Inc, ${ }^{\mathrm{h}}$ Alachua, Fla.

Disclosures: Dr Wang owns stock and is an executive officer of Banyan Biomarkers, Inc, and as such may benefit financially as a result of the outcomes of this research or the work reported in this publication. This study was supported by the National Institutes of Health (NIH RO1 HL091541-18 WAB and 1T32 CA126607-01A2 to G.J.A.). Drs Arnaoutakis and Weiss are the Irene Piccinini Investigators in Cardiac Surgery and Drs George and Allen are the Hugh R. Sharp Cardiac Surgery Research Fellows.

Read at the 91st Annual Meeting of The American Association for Thoracic Surgery, Philadelphia, Pennsylvania, May 7-11, 2011.

Received for publication May 6, 2011; revisions received June 12, 2011; accepted for publication June 28, 2011.

Address for reprints: William A. Baumgartner, MD, The Vincent L. Gott Professor of Surgery, Division of Cardiac Surgery, Department of Surgery, The Johns Hopkins University School of Medicine, Blalock 618, The Johns Hopkins Hospital, $600 \mathrm{~N}$ Wolfe St, Baltimore, MD 21287 (E-mail: wbaumgar@jhmi.edu).

0022-5223/\$36.00

Copyright (c) 2011 by The American Association for Thoracic Surgery doi:10.1016/j.jtcvs.2011.06.027
Despite advances in operative and anesthetic techniques, neurologic injury remains a devastating problem after cardiac surgery, with stroke rates between $1 \%$ and $6 \%{ }^{1,2}$ Furthermore, delirium after coronary artery bypass increases the risk of long-term mortality, ${ }^{3}$ and cognitive dysfunction is present in nearly one quarter of patients at 1 month. ${ }^{4}$ Patients with neurologic injury have increased hospital length of stay, cost, and need for inpatient rehabilitation. ${ }^{2}$ Although the risk of stroke remains relatively low, with nearly 300,000 cardiac surgical procedures performed annually in the United States, ${ }^{5}$ neurologic injury is a significant burden to patients, their families, and the health care system.

Nearly 35 years have elapsed since hypothermic circulatory arrest (HCA) was first introduced for cerebral protection during aortic arch reconstructions. ${ }^{6}$ Yet, end organ damage remains a significant problem in the setting of HCA, and there are no currently proven strategies to prevent brain injury. ${ }^{7,8}$ In the evolution of the surgical approach to conditions that require temporary cerebral blood flow exclusion, some centers have studied selective antegrade perfusion or retrograde cerebral perfusion. ${ }^{9,10}$ However, 


\section{Abbreviations and Acronyms \\ $\mathrm{CNS}=$ central nervous system \\ $\mathrm{CPB}=$ cardiopulmonary bypass \\ CSF $=$ cerebrospinal fluid \\ ELISA $=$ enzyme-linked immunosorbent assay \\ HCA = hypothermic circulatory arrest \\ UCHL1 = ubiquitin carboxyl-terminal esterase- L1}

HCA remains an established and viable strategy for complex aortic arch repairs and congenital cardiac lesions and is still widely used at many centers. ${ }^{8,11,12}$ Experimental evidence has implicated post-HCA glutamate excitotoxicity as an important mediator of this injury. ${ }^{13}$ Differential gene regulation has been detected in dogs undergoing HCA as compared with cardiopulmonary bypass (CPB) only, further elucidating the mechanisms in HCA-related brain injury. ${ }^{14}$

Brain injury is often diagnosed several hours to days after surgery; detecting neurologic injury early after the initial insult can enable clinicians to diagnose and implement treatments to reverse or mitigate the damage. Thus, the development of readily obtainable biomarkers that correlate with early brain injury harbors much promise for improving neurology related morbidity after cardiac surgery. Several biomarkers for brain injury have been widely studied, such as neuron-specific enolase, glial protein S-100 beta), and myelin basic protein; however, none has been definitively correlated with clinical outcomes, and studies relating these proteins to pathophysiologic parameters are lacking. ${ }^{15}$ Our laboratory detected spectrin breakdown products as biomarkers for central nervous system (CNS) injury, although these findings were confined to the cerebrospinal fluid (CSF). ${ }^{16}$

Because of abundant expression in neurons, ubiquitin carboxyl-terminal esterase-L1 (UCHL1) (first recognized as neuron-specific gene product 9.5$)^{17}$ has garnered enthusiasm as a potential CNS biomarker. UCHL1 accounts for nearly $5 \%$ of total soluble brain protein, is involved in ubiquitination pathways, and has been linked with neurodegenerative disorders such as Parkinson disease in humans. ${ }^{18}$ Recent evidence supports the detectable presence of UCHL1 in CSF in animal and human studies of subarachnoid hemorrhage and ischemic and traumatic brain injury. ${ }^{19-22}$ To date, there is only 1 study examining UCHL1 elevations after cardiac surgery, but those results were limited to CSF samples. ${ }^{23}$ Given the mounting evidence for UCHL1 as a biomarker for neuronal injury, we tested the hypothesis that UCHL1 is an effective serum biomarker for brain injury in a canine model of $\mathrm{CPB}$ and HCA.

\section{METHODS}

\section{Animals}

We used our clinically relevant canine model of HCA and CPB. ${ }^{13,14,16,24,25}$ Six- to 12-month-old, 30-kg male class A dogs were used (Marshal Bioresources, North Rose, NY). The Johns Hopkins Animal Care and Use Committee approved the experimental protocols, which complied with the "Guide for the Care and Use of Laboratory Animals" (1996, US National Institutes of Health).

\section{Experimental Design}

Dogs were randomly exposed to 2 hours of HCA ( $2 \mathrm{~h}-\mathrm{HCA} ; \mathrm{n}=20), 1$ hour of HCA (1h-HCA; $n=11)$, or CPB alone $(n=14)$ and survived to either 8 hours ( $2 \mathrm{~h}-\mathrm{HCA}, \mathrm{n}=10$; $1 \mathrm{~h}-\mathrm{HCA}, \mathrm{n}=5$; and $\mathrm{CPB}, \mathrm{n}=8$ ) or 24 hours ( $2 \mathrm{~h}-\mathrm{HCA}, \mathrm{n}=10 ; 1 \mathrm{~h}-\mathrm{HCA}, \mathrm{n}=6$; and $\mathrm{CPB}, \mathrm{n}=6$ ) after treatment. CSF and serum samples were collected at baseline (before the surgical incision but after induction of anesthesia), at 8 hours, and 24 hours. For both baseline and subsequent CSF collection, under sedation and in a routine sterile fashion, the spinal canal is entered with a 22-gauge needle through the cisterna magnum (at the base of the skull posteriorly). Samples are immediately frozen in a $-80^{\circ} \mathrm{C}$ freezer. Blood samples are obtained through previously placed peripheral intravenous catheters, cold centrifuged to collect serum, and frozen at $-80^{\circ} \mathrm{C}$. At the conclusion of the experiment, all subjects were humanely killed by exsanguination, and brains were harvested for analysis.

Surgical HCA procedure. Anesthesia was induced with methohexital sodium $9 \mathrm{mg} / \mathrm{kg}$. Animals were endotracheally intubated and maintained on inhaled isoflurane $(0.5 \%-2 \%), 100 \%$ oxygen, and intravenous fentanyl (150-200 $\mu \mathrm{g} /$ dose). Tympanic membrane, esophageal, and rectal probes monitored temperatures throughout the experiment. A left femoral artery cannula was placed for arterial blood gas and hemodynamic monitoring.

Standard CPB circuits with a 40- $\mu \mathrm{m}$ arterial filter (Sorin Group, Arvada, Colo) were used in all experiments. Intravenous heparin (300U/kg) was administered and the right femoral artery cannulated (12F-14F), advancing the cannula into the abdominal aorta. Two separate venous cannulas $(18 \mathrm{~F}-20 \mathrm{~F})$ were advanced to the right atrium via the right femoral and external jugular veins. Vessels were cannulated by an open cutdown technique. Closed-chest CPB was initiated using pump flows of 60 to $80 \mathrm{~mL} \cdot \mathrm{kg}^{-1} \cdot \mathrm{min}^{-1}$ to maintain mean arterial pressure of 60 to $80 \mathrm{~mm}$ $\mathrm{Hg}$, and activated clotting times were maintained greater than 500 seconds. For animals in the $1 \mathrm{~h}-\mathrm{HCA}$ or $2 \mathrm{~h}-\mathrm{HCA}$ groups, the pump was stopped when tympanic temperatures reached $18^{\circ} \mathrm{C}$ (approximately 30 minutes).

Animals underwent $1 \mathrm{~h}-\mathrm{HCA}$ or $2 \mathrm{~h}-\mathrm{HCA}$ with alpha-stat regulation of arterial blood gases $(\mathrm{pH}, 7.3-7.4$; arterial partial pressure of oxygen $>$ $300 \mathrm{~mm} \mathrm{Hg}$; and carbon dioxide, 30-40 mm Hg). Once HCA finished, $\mathrm{CPB}$ was resumed and rewarming commenced $\left(5^{\circ} \mathrm{C}\right.$ temperature gradient every 15 minutes to a core temperature of $37^{\circ} \mathrm{C}$ for 2 hours). Intravenous phenylephrine was used when necessary to maintain mean arterial pressure greater than $75 \mathrm{~mm} \mathrm{Hg}$. External defibrillation was performed when temperatures were $32^{\circ} \mathrm{C}$. At $37^{\circ} \mathrm{C}$, animals were separated from $\mathrm{CPB}$, decannulated, and reversed with protamine $(3 \mathrm{mg} / \mathrm{kg}$ intravenous).

Animals recovered from anesthesia while intubated, with frequent monitoring of vital signs, arterial blood gases, and urine output. Once hemodynamically and clinically stable, they were extubated and transferred to their crate for recovery. Analgesics were administered per protocol after the procedure.

CPB only. After induction and cannulation, animals underwent 2 hours of $\mathrm{CPB}$ without HCA. Animals were cooled to $32^{\circ} \mathrm{C}$, and the heart continued to beat during this operation. Animals were allowed to recover from anesthesia as described above.

Neurologic assessment. The University of Pittsburgh Canine Neurological Score was independently determined at 24 hours by 2 nonblinded 
study team members. ${ }^{26}$ The score includes 22 clinical questions relating to level of consciousness, respiration, cranial nerve function, reflexes, behavior, and motor and sensory function. Animals had normal neurologic function before experimentation. No additional sedation was given within 12 hours of neurologic assessment.

Humane death and tissue procurement. Animals were sedated, intubated, and anesthetized. A sternotomy was performed, 300 units $/ \mathrm{kg}$ of heparin given, and the ascending aorta cannulated (22F). Cold perfusion via the aortic cannula flushed the brain with $12 \mathrm{~L}$ of saline solution $\left(4^{\circ} \mathrm{C}\right)$ at $60 \mathrm{~mm} \mathrm{Hg}$. The right atrial appendage was transected and venous return suctioned into a reservoir. Brains were harvested by a wide craniectomy.

Tissue preparation and UCHL1 immunohistochemistry. Brains were immersion-fixed for 1 week in $4 \%$ paraformaldehyde, cut into 2-mm coronal blocks, embedded in paraffin, and sectioned at $6 \mu \mathrm{m}$. For immunohistochemistry, endogenous peroxidase was quenched (3\% hydrogen peroxide in methanol, 10 minutes, room temperature), slides were blocked (phosphate-buffered saline with 5\% normal goat serum, $0.2 \%$ Triton $\mathrm{X}$ 100 [Union Carbide Corporation, Danbury, Conn], and 0.2\% gelatin, 60 minutes, RT) and incubated in rabbit anti-UCHL1 (Novus Biologicals NB100-65827 [Novus Biologicals, Littleton, Colo], 1:1600 in phosphatebuffered saline with $1.5 \%$ normal goat serum, $0.2 \%$ Triton $\mathrm{X}-100,0.2 \%$ gelatin, and $1 \mu \mathrm{L} / \mathrm{mL}$ sodium azide, 48 hours, $4^{\circ} \mathrm{C}$ ). The primary antibody was visualized using the avidin-biotin-peroxidase complex method (ABCElite; Vector Laboratories, Inc, Burlingame, Calif).

\section{Histologic Analysis}

Hematoxylin and eosin staining was performed for blinded histologic evaluation by a single neuropathologist (J.C.T.). Eleven distinct regions of the canine brain were evaluated for the presence of apoptosis and necrosis. These regions include midfrontal cortex, superior parietal cortex, basal ganglia, hippocampus (dentate gyrus and CA regions), entorhinal cortex, amygdala, cerebellum (molecular layer, Purkinje layer, and granule layer), and brainstem. A semiquantitative scale was used to assess the degree of necrosis and apoptosis in each region. These scores were summed to obtain the neuronal cell death score, ranging from a minimum of 0 (no damage) to a maximum of 99 (extreme neuronal damage).

\section{ELISA Analysis}

UCHL1 levels in CSF and serum were measured using a UCHL1 sandwich enzyme-linked immunosorbent assay (ELISA) modified from a protocol previously reported. ${ }^{20,21}$ Mouse monoclonal antihuman UCHL1 antibody and rabbit polyclonal antihuman UCHL1 antibody were made in-house against recombinant human UCHL1 full-length protein and partial protein, respectively. Both were affinity purified and specificity was confirmed by immunoblotting. Reaction wells were coated with capture antibody (purified mouse monoclonal antihuman UCHL1) in 0.05-mol/L sodium bicarbonate, $\mathrm{pH} 9.6$, and incubated overnight at $4{ }^{\circ} \mathrm{C}$. Plates were then washed with blocking buffer (Tris buffer saline with $0.02 \%$ Tween-20 [volume/volume]) and further incubated for 30 minutes at ambient temperature with gentle shaking. Antigen standard (UCHL1 standard curve, 0, 0.06-15 $\mathrm{ng} / \mathrm{mL}$ ), unknown samples ( $5 \mu \mathrm{L}$ of CSF; $20 \mu \mathrm{L}$ of serum), or assay internal control samples were incubated overnight with detection antibody (rabbit polyclonal antihuman UCHL1, $100 \mu \mathrm{L}$ total volume). The capture antibody-coated plate was then incubated with detection antibody sample mixture for 1.5 hours at room temperature and washed using an automatic plate washer (each well rinsed with $350 \mu \mathrm{L}$ Tris/Tween-20 wash buffer). The plate was then incubated with antirabbit-immunoglobulin G-horseradish peroxidase (Amersham Biosciences, Buckinghamshire, United Kingdom) at room temperature for 1 hour and developed with UltraTMB ELISA substrate (Pierce No. 34028; Thermo Scientific Pierce Protein Research Products, Rockford, Ill) for 10 minutes. The plate was read at 450 $\mathrm{nm}$ with a Molecular Devices Spectramax 190 spectrophotometer
(Molecular Devices, Inc, Sunnyvale, Calif). The intra-assay coefficient of variance equaled $2.1 \%$ to $7.9 \%$ whereas the interassay coefficient of variance equaled $0.9 \%$ to $10.6 \%$ within the assay dynamic range. The limit of detection was $0.030 \mathrm{ng} / \mathrm{mL}$; samples with undetectable levels were assigned $50 \%$ of the limit of detection (ie, $0.015 \mathrm{ng} / \mathrm{mL}$ ). If samples yielded a signal above the quantification range, samples were diluted and reassayed. For all serum samples, concentrations were normalized against total serum protein concentration for the same sample ( $\mathrm{ng} / \mathrm{mg}$ protein).

\section{Genomic Analysis}

Canine microarray analysis was performed in a blinded fashion at the Johns Hopkins Deep Sequencing and Microarray Core Facility. Detailed methods of this protocol have been described previously. ${ }^{14}$ Gene expression profiles in samples from ventral anterior hippocampus were compared between 2h-HCA, 1h-HCA, CPB, and untreated normal dogs (each treatment group was compared with normal controls). Exploratory data analysis was performed on normalized data with a false discovery rate less than 0.10 considered significantly regulated. ${ }^{27}$ Entrez Gene IDs for human orthologs were assigned to evaluate gene function, and Ingenuity Pathways Analysis software (Ingenuity Systems, Inc, http://www. ingenuity.com, Redwood City, Calif) was used to identify proteins that interact directly with UCHL1 or are part of canonical pathways that include UCHL1. For UCHL1-related genes that were significantly regulated in one or more treatment groups, fold change versus normal was determined in all groups.

\section{Statistical Analysis}

Neurologic scores are presented as mean \pm standard deviation. Oneway analysis of variance compared neurologic and histologic scores among groups. For all subjects, the paired comparisons $t$ test examined differences in levels of UCHL1 from baseline to 8 hours after treatment. For animals surviving 24 hours, repeated-measures analysis of variance was used to account for the repeated serum samples from within the same canine subject over time. Post hoc pairwise comparisons were conducted using the Tukey-Kramer method. Correlations between functional and histopathologic scores were assessed using linear regression and the Pearson correlation coefficient. Threshold values for UCHL1 levels that predict severe functional impairment (neurologic score $>100$ ) were determined using unadjusted logistic regression and receiver operating characteristic curves (area under the curve $>0.7$ considered significant). Analysis was performed using STATA software (version 9.2; StataCorpLP, College Station, Tex).

\section{RESULTS \\ Subjects}

Twenty dogs underwent 2h-HCA, 11 had $1 \mathrm{~h}-\mathrm{HCA}$, and 14 had CPB. Roughly half of the dogs in each group ( $2 \mathrm{~h}-$ $\mathrm{HCA}, \mathrm{n}=10$; $1 \mathrm{~h}-\mathrm{HCA}, \mathrm{n}=5$; and $\mathrm{CPB}, \mathrm{n}=8$ ) were humanely killed at 8 hours and the other half at 24 hours.

\section{Neurologic Scores}

Using the neurologic scoring system (0-480), higher scores indicate worse neurologic function. Scores at 24 hours were significantly different among groups, with lower scores observed in groups subjected to milder insult (Figure 1). For 2h-HCA animals, the mean neurologic score was $185 \pm 28$. In $1 \mathrm{~h}$-HCA animals, the mean score was 40 \pm 50 . Two 1h-HCA dogs were effectively normal (scores $<20$ ); in contrast, no $2 \mathrm{~h}-\mathrm{HCA}$ dog had a score less than 130: all were severely impaired. CPB animals 


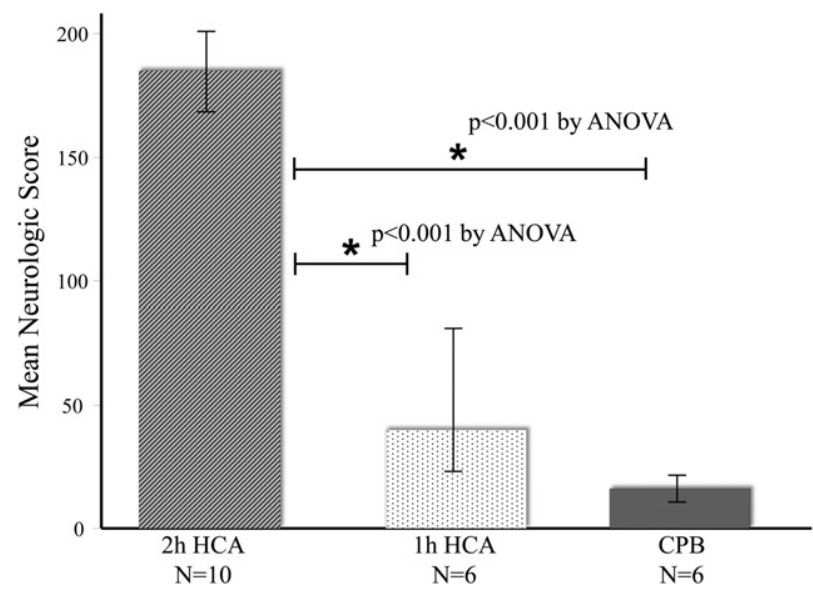

FIGURE 1. Average neurologic scores (based on 2 independent observers) for animals 24 hours after $2 \mathrm{~h}-\mathrm{HCA}, 1 \mathrm{~h}-\mathrm{HCA}$, or CPB, using the University of Pittsburgh Canine Neurological Score (0-480, with higher scores corresponding with greater functional neurologic impairment). *Indicates $P<.05$ by Tukey-Kramer method post hoc pairwise comparisons. $A N O V A$, Analysis of variance; $H C A$, hypothermic circulatory arrest; $C P B$, cardiopulmonary bypass; $h$, hours.

demonstrated negligible change in neurologic function (mean score, $16 \pm 16$ ).

\section{Histopathology Scores}

Dogs exposed to 2h-HCA had the highest levels of cellular injury on histologic examination (mean neuronal cell death score, $9.6 \pm 5.5$; Table 1). This level was different when compared with 1h-HCA and CPB groups. Most brain regions showed minimal neuronal death, but at 24 hours in the $2 \mathrm{~h}$-HCA group the hippocampus was severely affected (up to $75 \%$ apoptotic neurons in the dentate gyrus). Linear regression analysis showed a strong correlation between histopathologic scores and University of Pittsburgh Functional Assessment scores (Figure 2).

\section{Immunohistochemistry Results}

UCHL1 staining in the hippocampus was less in $2 \mathrm{~h}-\mathrm{HCA}$ animals than in 1h-HCA and CPB subjects. In the dentate gyrus, there was moderate expression of UCHL1 in the CPB group but almost complete loss of protein expression in the $2 \mathrm{~h}$-HCA group. In the $\mathrm{CA} 3$ region, there was a change from high expression in the $\mathrm{CPB}$ group to moderate expression in pyramidal neurons after $2 \mathrm{~h}-\mathrm{HCA}$. However, there was near-complete loss of UCHL1 in the mossy fiber layer after $2 \mathrm{~h}$-HCA, where axon terminals from the depleted dentate granule neurons are located (Figure 3).

\section{ELISA Results}

Serum. In $2 \mathrm{~h}-\mathrm{HCA}$ animals, serum UCHL1 levels were increased from baseline 8 hours after treatment $(3.2 \pm 0.3 \mathrm{ng} /$ $\mathrm{mg}$ protein to $9.3 \pm 2.0 \mathrm{ng} / \mathrm{mg}$ protein; $P=.01$ ) but were similar to baseline by 24 hours (Figure $4, A$ ). In 1h-HCA and CPB animals, serum levels of UCHL1 were not elevated at either time point. Comparison of the 3 groups revealed that $2 \mathrm{~h}$-HCA animals had significantly elevated UCHL1 in serum at 8 hours compared with CPB $(P=.01)$, but by 24 hours there were no longer any detectable differences among the groups. Correlation of serum UCHL1 levels for the 3 groups against University of Pittsburgh Functional Assessment scores revealed that $3.9 \mathrm{ng} /$ mg total protein appeared the most sensitive break point for predicting functional impairment in an unadjusted logistic regression analysis (area under receiver operating characteristic curve, $0.9 ; P=.02$ ) (Figure $4, B$ ).

CSF. In 2h-HCA animals, UCHL1 protein in CSF was significantly increased from baseline 8 hours after HCA $(11.7 \pm 6.0 \mathrm{ng} / \mathrm{mL}$ to $144.5 \pm 21.7 \mathrm{ng} / \mathrm{mL} ; P<.01)$ and remained elevated 24 hours after HCA $(P<.01)$. In 1 hHCA and CPB animals, UCHL1 levels were elevated at 8 hours compared with baseline (1h-HCA, $5.4 \pm 2.4$ $\mathrm{ng} / \mathrm{mL}$ to $56.2 \pm 18.6 \mathrm{ng} / \mathrm{mL} ; P=.01 ; \mathrm{CPB}, 12.4 \pm 6.1$ $\mathrm{ng} / \mathrm{mL}$ to $68.1 \pm 21.0 \mathrm{ng} / \mathrm{mL} ; P=.02$ ) but returned to baseline levels at 24 hours. Comparison among the 3 groups at 8 and 24 hours revealed that $2 \mathrm{~h}$-HCA animals had elevated levels of UCHL1 compared with 1h-HCA and $\mathrm{CPB}$ animals at both time points (Figure E1, A). CSF levels of UCHL1 for the 3 groups were correlated with University of Pittsburgh Functional Assessment scores. The most sensitive break point for predicting functional impairment in an unadjusted logistic regression analysis was $61 \mathrm{ng} / \mathrm{mL}$ (receiver operating characteristic curve area, 0.77; $P=.09$ ) (Figure E1, $B$ ).

TABLE 1. Average histologic scores for neuronal cell death (total and by brain region)

\begin{tabular}{lccccc}
\hline \multicolumn{1}{c}{ Brain region } & Potential score & 2h-HCA $(\mathbf{n}=\mathbf{1 0})$ & 1h-HCA $(\mathbf{n}=\mathbf{6})$ & CPB $(\mathbf{n}=\mathbf{6})$ & $\boldsymbol{P}$ value* \\
\hline Composite score of all brain regions examined & 99 & $9.6( \pm 5.5) \dagger, \ddagger$ & $4.1( \pm 2.2)$ & $0.7( \pm 1.3)$ \\
Notable regions & & & & \\
$\quad$ Cortex & 18 & $1.3( \pm 1.4) \dagger, \ddagger$ & $0.5( \pm 0.7)$ & $0( \pm 0)$ \\
Basal ganglia & 9 & $1.0( \pm 0.5) \dagger, \ddagger$ & $0.6( \pm 0.6)$ & $0.1( \pm 0.3)$ & $<.02$ \\
Hippocampus & 18 & $3.2( \pm 0.9) \dagger, \ddagger$ & $1.3( \pm 0.9) \dagger$ & $0.1( \pm 0.3)$ & $<.01$ \\
Cerebellum & 27 & $0.9( \pm 0.9) \S$ & $0.9( \pm 0.8)$ & $0.1( \pm 0.4)$ & .14 \\
\hline
\end{tabular}

$H C A$, Hypothermic circulatory arrest; $C P B$, cardiopulmonary bypass. Eleven distinct brain regions examined included the following: midfrontal cortex, superior parietal cortex, basal ganglia, hippocampus (dentate gyrus and CA region), entorhinal cortex, amygdala, cerebellum (molecular layer, Purkinje layer, and granule layer), and brainstem. ${ }^{*} P$ value

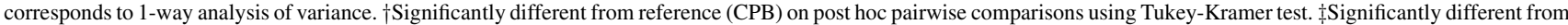
1h-HCA on post-hoc pairwise using Tukey-Kramer test. §2h-HCA subjects demonstrate excessive hemorrhage on histologic analysis. 


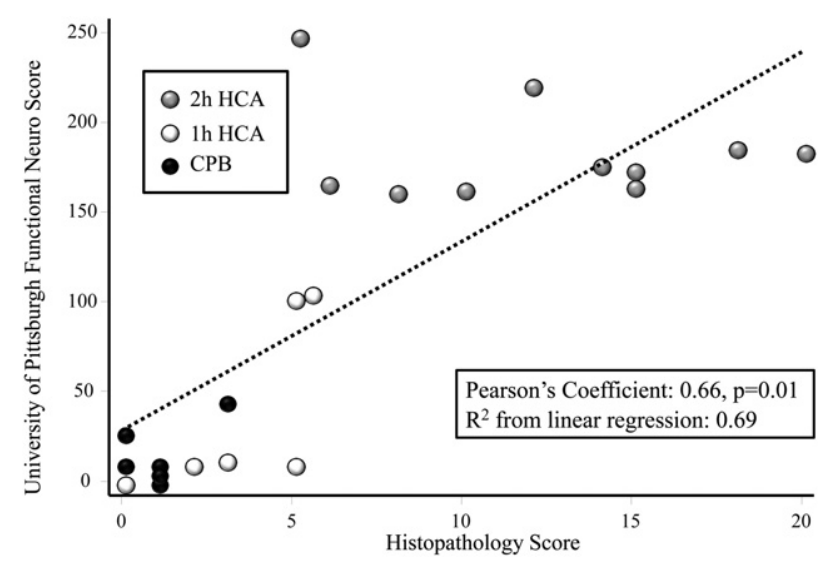

FIGURE 2. Linear regression analysis depicting correlation between histopathology scores and University of Pittsburgh Functional Assessment Scores. Strength of correlation was determined using linear regression analysis with the Pearson coefficient and $R^{2}$ value. HCA, Hypothermic circulatory arrest; $C P B$, cardiopulmonary bypass.

\section{Genomic Results}

In $2 \mathrm{~h}$-HCA animals, 3 genes were significantly regulated at 8 hours, and 8 genes were significantly regulated at 24 hours. In the 1h-HCA group, 1 gene was significantly regulated at 8 hours and none at 24 hours. No genes were significantly regulated in the $\mathrm{CPB}$ group. Among the regulated genes, 3 messenger RNAs for proteins that directly interact with UCHL1 and 2 messenger RNAs for proteins in the Parkin pathway were significantly downregulated, but 6 messenger RNAs for proteasome subunits were significantly upregulated. Detailed information regarding regulated genes is shown in Table E1.

\section{DISCUSSION}

The promising field of proteomics has facilitated the development of new biomarkers specific for organ injury. The advantages of neuron-specific biomarkers are threefold: Earlier detection of brain injury may (1) allow for therapeutic, pharmacologic, and hemodynamic
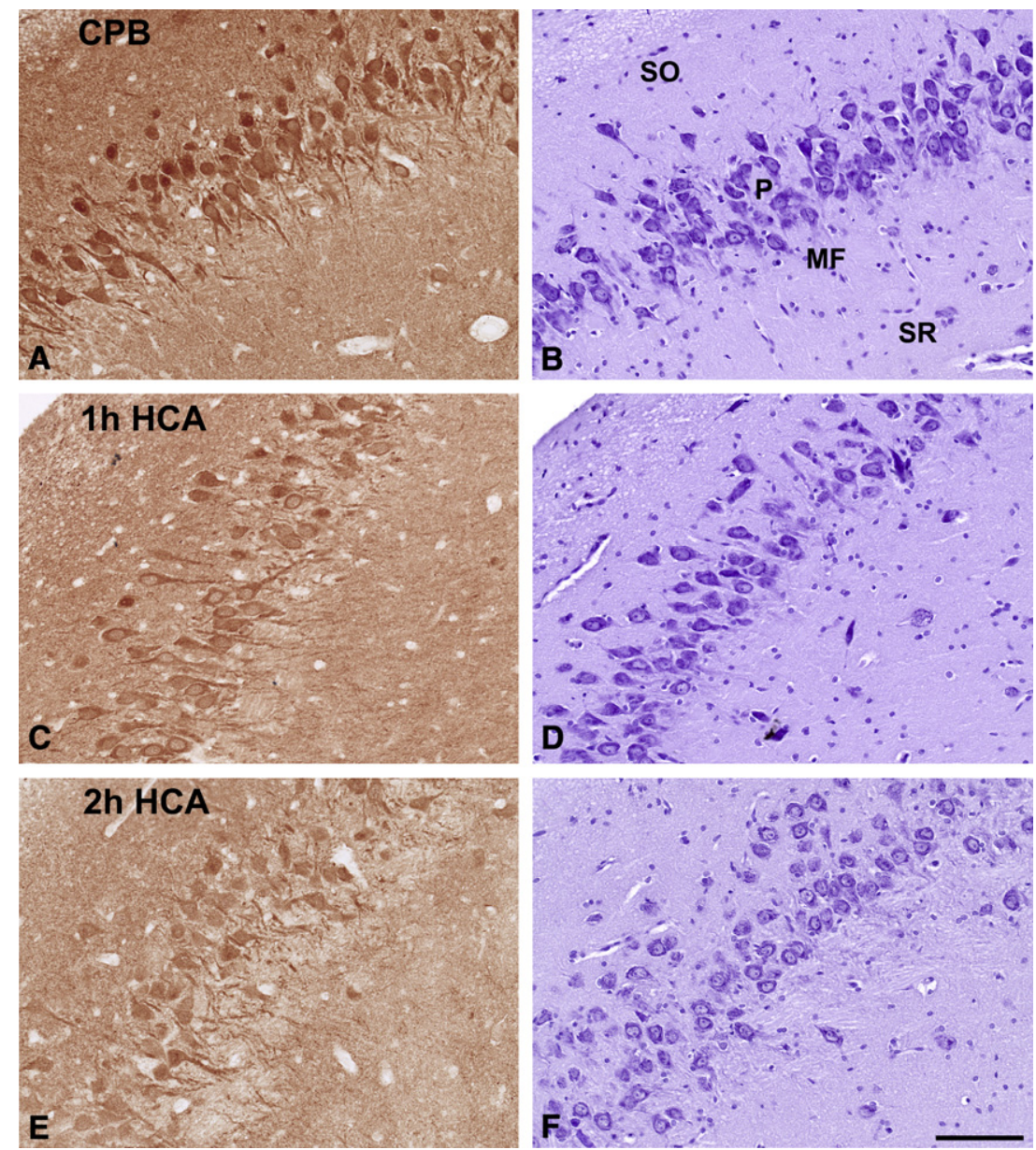

FIGURE 3. UCHL1 immunoreactivity (left panel) and corresponding Nissl-stained sections (right panel) in CA3 region of hippocampus for CPB (A, B), 1h-HCA (C, D), and 2h-HCA (E, F). UCHL1, Ubiquitin carboxyl-terminal esterase-L1; HCA, hypothermic circulatory arrest; $C P B$, cardiopulmonary bypass; $S O$, stratum oriens; $P$, pyramidal neurons; $M F$, mossy fiber layer; $S R$, stratum radiatum. 


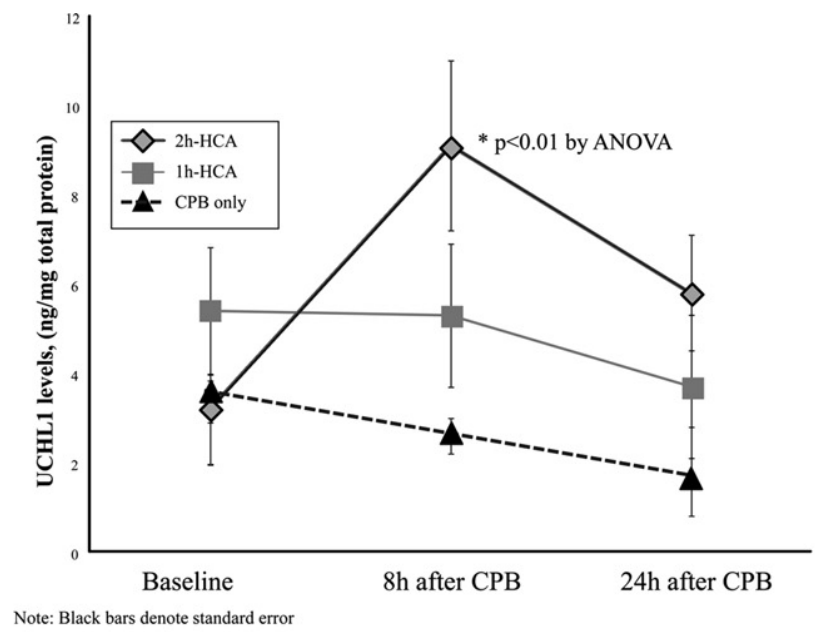

A

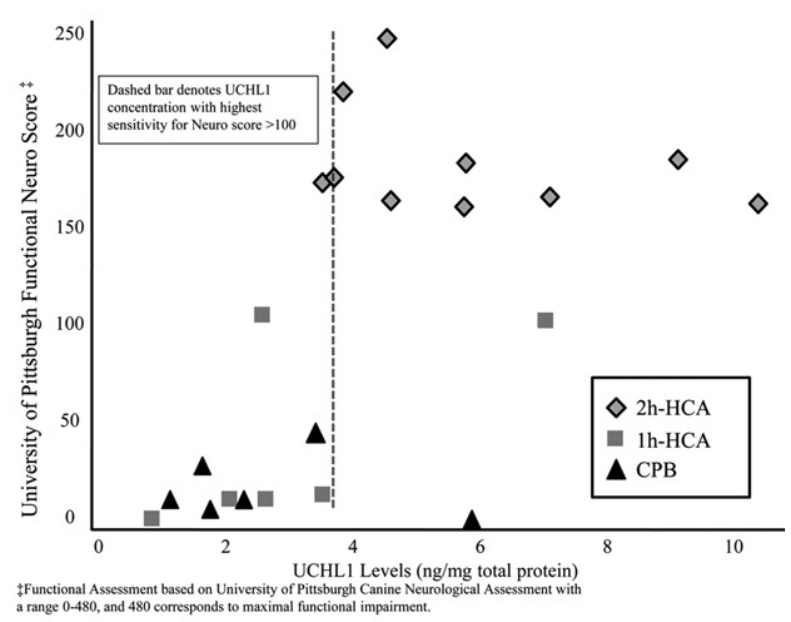

B

FIGURE 4. Line graph (A) showing serum levels of UCHL1 by treatment groups across 3 time points. Scatter plots (B) for serum UCHL1 levels correlated with functional assessment score. Break point denoted by dashed line indicates highest sensitivity for predicting outcome of interest (Neuro Score $>100$ ) by univariate logistic regression analysis. ANOVA, Analysis of variance; $H C A$, hypothermic circulatory arrest; $C P B$, cardiopulmonary bypass; $U C H L 1$, ubiquitin carboxyl-terminal esterase-L1.

interventions, (2) improve prognostic abilities, and (3) aid in future research. UCHL1 is a candidate serum biomarker known to be abundant with high specificity in neurons. ${ }^{17,26}$ It has further been linked with several neurodegenerative diseases in humans, including Parkinson disease. ${ }^{18}$ Dogs undergoing prolonged HCA ( 2 hours) had significant increases in serum UCHL1 protein at 8 hours compared with baseline and compared with the $\mathrm{CPB}$ group at 8 hours. The $2 \mathrm{~h}$-HCA dogs also had greater neurologic impairment and histologic damage. Immunohistochemistry analysis elucidated plausible biologic mechanisms to support the ELISA results, and genomic analysis suggests downregulation of proteins that bind to UCHL1 and possible compensatory upregulation of proteasomal subunits. Taken collectively, these findings support the use of UCHL1 as a serum biomarker specific for neurologic injury after HCA.

Consistent with our earlier work, dogs subjected to $2 \mathrm{~h}$ HCA sustained significantly greater histologic damage (most pronounced in the hippocampus) compared with those subjected to 1h-HCA and CPB only. Because our purpose was to assess the utility of the UCHL1 biomarker as an indicator of clinical brain injury, we used the University of Pittsburgh Functional Assessment score (cutoff $>100$ ) as our dependent variable in unadjusted logistic regression analysis. This scoring system is a validated instrument for this purpose, and regression analysis confirmed a reliable correlation between this measure of neurologic impairment and histopathologic scores (Figure 2) ${ }^{26}$ This supports our decision to use the functional assessment score as the outcome measure; however, this outcome measure was determined only at 24 hours of survival.
The immunohistochemistry results substantiate the elevated levels of UCHL1 detected in the CSF and serum. ICH staining revealed a progressive loss of neuronal UCHL1 protein with increasing severity of treatment, which is consistent with the progressive increase observed in CSF. The brains of animals subjected to CPB had preserved UCHL1 immunoreactivity within intact neurons. This staining was less intense for $1 \mathrm{~h}-\mathrm{HCA}$ dogs and greatly diminished in the 2h-HCA group. Consistent with this finding, 11 genes intimately related to UCHL1 were upregulated or downregulated after $2 \mathrm{~h}-\mathrm{HCA}$; in comparison, only 1 gene was significantly regulated in $1 \mathrm{~h}-\mathrm{HCA}$ and none after CPB only. This transcriptional profile across the 3 treatment groups mirrors our earlier findings on transcriptional regulation among a broad array of gene categories and supports the safety of CPB.${ }^{14}$ In this study, we elected to use Ingenuity Pathways Analysis to identify genes related to UCHL1. This system is human-curated and, therefore, only validated interactions and plausible mechanistic pathways are considered.

We detected elevations of UCHL1 in the CSF at 8 hours among all 3 study groups. However, serum elevations of UCHL1 were observed only in the $2 \mathrm{~h}$-HCA group at 8 hours. So that sensitivity and specificity will be enhanced in clinical use, a panel of biomarkers with different time course profiles for release and degradation will be important, akin to the use of serum markers for myocardial infarction. Although the sandwich ELISA yields highly quantitative results, we applied rigorous methods to standardize the serum concentrations by using the total protein concentration for the respective sample. Thus, we believe the serum results are robust and valid. 
The immunohistochemistry analysis supports the serum findings, demonstrating release of UCHL1 protein from injured neurons in the 2h-HCA group. Although UCHL1 release is believed to be associated with neuronal injury/ death, we believe that the early presence of this biomarker may aid in identification of threatened yet salvageable neurons in adjacent regions of the brain (after intraoperative or perioperative hypoperfusion or watershed infarctions). Pharmacologic interventions, as well as aggressive treatment of hypoxia and hypotension, may spare these threatened neurons. Future clinical studies are needed to fully assess the ability of this marker to influence such therapies.

\section{PREVIOUS WORK}

Our laboratory has previously reported the elevations of spectrin breakdown products in the CSF after 1h-HCA. ${ }^{16}$ A novel contribution of the current study is the detection of a novel biomarker specific for CNS injury in serum. Additional studies have investigated the role of UCHL1 as a biomarker in the setting of traumatic brain injury, subarachnoid hemorrhage, and ischemia-reperfusion injury. ${ }^{19-21,23}$ Papa and associates ${ }^{21}$ detected higher CSF levels of UCHL1 in 44 human patients who had sustained traumatic brain injury. That study examined additional time points, with detailed characterization of the time course of CSF UCHL1 protein levels; however, the mechanism of neurologic injury differs from HCA. Lewis and colleagues $^{19}$ reported 30 patients with elevations of CSF UCHL1 after subarachnoid hemorrhage and observed higher mortality rates among patients with the highest levels of UCHL1. Siman and coworkers ${ }^{23}$ conducted the only study to date conducted in cardiac surgery patients, with all 19 patients undergoing thoracic aortic aneurysm repair, 7 of whom required HCA. Among the 12 patients not requiring HCA (aortic crossclamping only), patients with acute neurologic complications had higher CSF UCHL1 levels, suggesting UCHL1 release in response to warm ischemia and reperfusion injury. This study did not, however, examine serum levels of UCHL1. Our study builds on these existing data by identifying serum elevations of UCHL1 associated with neurologic injury in the setting of HCA.

\section{LIMITATIONS}

This study is limited with respect to the temporal pattern of biomarker release into the CSF and serum. The current data suggest that UCHL1 peaks in the serum 8 hours after HCA and begins to lower by 24 hours. However, it is possible that there is an earlier peak. We acknowledge this limitation; however, we attempted to balance study feasibility with prior experience regarding the temporal release of UCHL1 after neurologic injury. Additional studies of groups having shorter survival times are currently being conducted to address this important issue. This study also does not provide data regarding long-term or late release of this biomarker; such information can only be gleaned from longer survival models, which pose additional logistical obstacles. There are outliers within the data points, but these were not excluded to preserve the purity of the data set. Also, when the relationship between functional impairment and serum UCHL1 concentrations within individual treatment groups is examined (Figure 4, $B$ ), there does not appear to be a positive correlation. We speculate that a larger sample size and more sensitive functional assessment (as can be performed in humans) would address these limitations.

We were unable to control for the effects of hypothermia on UCHL1 release. Hypothermia may mediate pathways involved in the release of UCHL1. However, given prior experience suggesting a protective effect of hypothermia on neurologic function ${ }^{28}$ and the preponderance of evidence suggesting that UCHL1 is released in response to neurologic injury, it is unlikely that the serum presence of UCHL1 observed in this study is due purely to hypothermia. Furthermore, it would be difficult to achieve such an experimental condition, inasmuch as all animals experience spontaneous ventricular fibrillation when undergoing controlled hypothermia.

From a logistical standpoint, if we were to have designed a comparison control group that was progressively cooled for 30 minutes to deep hypothermia and rewarmed afterward, our past experience suggests nearly all of these dogs would have had a period of ventricular fibrillation. The HCA groups are not subjected to a prolonged duration of ventricular fibrillation because the pump is turned off during the arrest phase. The groups would have been better matched with respect to the incidence of fibrillation (and the requirement of defibrillation); however, this hypothetical control group would have been exposed to a longer period of fibrillation.

Additionally, we acknowledge that a 2-hour period to induce injury is quite severe and does not mirror clinical practice. However, the dogs used in this study were all young, healthy animals without cerebrovascular disease or prior stroke. In the interest of efficient use of animals and resources, we chose to study an HCA duration that exceeded clinical practice but would ensure histologic damage and maximize the likelihood of detecting biomarker elevations in the serum.

\section{CONCLUSIONS}

In summary, this is the first study to document serum elevation of UCHL1 after neurologic injury. The novel neuronal biomarker UCHL1 is increased in serum 8 hours after severe neurologic insult in $2 \mathrm{~h}-\mathrm{HCA}$ animals compared with animals in the CPB group. These results support the potential for practical use in patients undergoing cardiac surgery and form the basis for clinical correlation in human studies. 
We thank Ms Jennifer Berrong for her expertise in brain tissue preparation. We also express profound gratitude to Mr Jeffrey Brawn and Mrs Melissa Jones for their invaluable technical assistance and support. Their decades-long experience with the experimental protocol was vital to completion of this study.

\section{References}

1. McKhann GM, Grega MA, Borowicz LM Jr, Bechamps M, Selnes OA, Baumgartner WA, et al. Encephalopathy and stroke after coronary artery bypass grafting: incidence, consequences, and prediction. Arch Neurol. 2002;59:1422-8.

2. Roach GW, Kanchuger M, Mangano CM, Newman M, Nussmeier N, Wolman R, et al. Adverse cerebral outcomes after coronary bypass surgery. Multicenter Study of Perioperative Ischemia Research Group and the Ischemia Research and Education Foundation Investigators. N Engl J Med. 1996;335:1857-63.

3. Gottesman RF, Grega MA, Bailey MM, Pham LD, Zeger SL, Baumgartner WA, et al. Delirium after coronary artery bypass graft surgery and late mortality. Ann Neurol. 2010;67:338-44

4. McKhann GM, Goldsborough MA, Borowicz LM Jr, Selnes OA, Mellits ED, Enger C, et al. Cognitive outcome after coronary artery bypass: a one-year prospective study. Ann Thorac Surg. 1997;63:510-5.

5. Society of Thoracic Surgeons. Society of Thoracic Surgeons National Database Executive Summary. Available at: http://www.sts.org/documents/pdf/ndb2010/ 1stHarvestExecutiveSummary\%5B1\%5D.pdf. Accessed January 1, 2011.

6. Griepp RB, Stinson EB, Hollingsworth JF, Buehler D. Prosthetic replacement of the aortic arch. J Thorac Cardiovasc Surg. 1975;70:1051-63.

7. Arnaoutakis GJ, Bihorac A, Martin TD, Hess PJ Jr, Klodell CT, Ejaz AA, et al. RIFLE criteria for acute kidney injury in aortic arch surgery. J Thorac Cardiovasc Surg. 2007;134:1554-60; discussion 1560-1.

8. Augoustides JG, Floyd TF, McGarvey ML, Ochroch EA, Pochettino A, Fulford S, et al. Major clinical outcomes in adults undergoing thoracic aortic surgery requiring deep hypothermic circulatory arrest: quantification of organ-based perioperative outcome and detection of opportunities for perioperative intervention. J Cardiothorac Vasc Anesth. 2005; 19:446-52.

9. Di Eusanio M, Schepens MA, Morshuis WJ, Di Bartolomeo R, Pierangeli A, Dossche KM. Antegrade selective cerebral perfusion during operations on the thoracic aorta: factors influencing survival and neurologic outcome in 413 patients. J Thorac Cardiovasc Surg. 2002;124:1080-6.

10. Lytle BW, McCarthy PM, Meaney KM, Stewart RW, Cosgrove DM 3rd. Systemic hypothermia and circulatory arrest combined with arterial perfusion of the superior vena cava: effective intraoperative cerebral protection. J Thorac Cardiovasc Surg. 1995;109:738-43.

11. Elefteriades JA. What is the best method for brain protection in surgery of the aortic arch? Straight DHCA. Cardiol Clin. 2010;28:381-7.

12. Gega A, Rizzo JA, Johnson MH, Tranquilli M, Farkas EA, Elefteriades JA. Straight deep hypothermic arrest: experience in 394 patients supports its effectiveness as a sole means of brain preservation. Ann Thorac Surg. 2007;84: 759-66; discussion 766-7

13. Williams JA, Barreiro CJ, Nwakanma LU, Lange MS, Kratz LE, Blue ME, et al. Valproic acid prevents brain injury in a canine model of hypothermic circulatory arrest: a promising new approach to neuroprotection during cardiac surgery. Ann Thorac Surg. 2006;81:2235-41; discussion 2241-2.

14. Allen JG, Weiss ES, Wilson MA, Arnaoutakis GJ, Blue ME, Talbot CC Jr, et al. Hawley H. Seiler Resident Award. Transcriptional profile of brain injury in hypothermic circulatory arrest and cardiopulmonary bypass. Ann Thorac Surg. 2010;89:1965-71.

15. Laterza OF, Modur VR, Crimmins DL, Olander JV, Landt Y, Lee JM, et al. Identification of novel brain biomarkers. Clin Chem. 2006;52:1713-21.

16. Weiss ES, Wang KK, Allen JG, Blue ME, Nwakanma LU, Liu MC, et al. Alpha IIspectrin breakdown products serve as novel markers of brain injury severity in a canine model of hypothermic circulatory arrest. Ann Thorac Surg. 2009;88:543-50.

17. Doran JF, Jackson P, Kynoch PA, Thompson RJ. Isolation of PGP 9.5, a new human neurone-specific protein detected by high-resolution two-dimensional electrophoresis. J Neurochem. 1983;40:1542-7.

18. Liu Z, Meray RK, Grammatopoulos TN, Fredenburg RA, Cookson MR, Liu Y, et al. Membrane-associated farnesylated UCH-L1 promotes alpha-synuclein neurotoxicity and is a therapeutic target for Parkinson's disease. Proc Natl Acad Sci U S A. 2009;106:4635-40.

19. Lewis SB, Wolper R, Chi YY, Miralia L, Wang Y, Yang C, et al. Identification and preliminary characterization of ubiquitin C terminal hydrolase 1 (UCHL1) as a biomarker of neuronal loss in aneurysmal subarachnoid hemorrhage. J Neurosci Res. 2010;88:1475-84.

20. Liu MC, Akinyi L, Scharf D, Mo J, Larner SF, Muller U, et al. Ubiquitin C-terminal hydrolase-L1 as a biomarker for ischemic and traumatic brain injury in rats. Eur J Neurosci. 2009;31:722-32.

21. Papa L, Akinyi L, Liu MC, Pineda JA, Tepas JJ 3rd, Oli MW, et al. Ubiquitin $\mathrm{C}$-terminal hydrolase is a novel biomarker in humans for severe traumatic brain injury. Crit Care Med. 2010;38:138-44.

22. Svetlov SI, Prima V, Kirk DR, Gutierrez H, Curley KC, Hayes RL, et al. Morphologic and biochemical characterization of brain injury in a model of controlled blast overpressure exposure. J Trauma. 2010;69:795-804.

23. Siman R, Roberts VL, McNeil E, Dang A, Bavaria JE, Ramchandren S, et al. Biomarker evidence for mild central nervous system injury after surgically-induced circulation arrest. Brain Res. 2008;1213:1-11.

24. Redmond JM, Gillinov AM, Zehr KJ, Blue ME, Troncoso JC, Reitz BA, et al. Glutamate excitotoxicity: a mechanism of neurologic injury associated with hypothermic circulatory arrest. J Thorac Cardiovasc Surg. 1994;107:776-86; discussion 786-787

25. Barreiro CJ, Williams JA, Fitton TP, Lange MS, Blue ME, Kratz L, et al. Noninvasive assessment of brain injury in a canine model of hypothermic circulatory arrest using magnetic resonance spectroscopy. Ann Thorac Surg. 2006;81:1593-8.

26. Tisherman SA, Safar P, Radovsky A, Peitzman A, Sterz F, Kuboyama K. Therapeutic deep hypothermic circulatory arrest in dogs: a resuscitation modality for hemorrhagic shock with "irreparable" injury. J Trauma. 1990;30: 836-47.

27. Benjamini Y, Hockberg Y. Controlling the false discovery rate: a practical and powerful approach to multiple testing. J R Stat Soc Series B Methodol. 1995; 57:289-300

28. Coselli JS, Crawford ES, Beall AC Jr, Mizrahi EM, Hess KR, Patel VM. Determination of brain temperatures for safe circulatory arrest during cardiovascular operation. Ann Thorac Surg. 1988;45:638-42.

\section{Discussion}

Dr J. William Gaynor (Philadelphia, Pa). I have 2 questions. First, is this marker expressed in tissues other than the brain? We have seen problems with some other markers such as S100 expressed in other tissues that can be injured. Second, what is the developmental expression? Obviously, I am interested in the immature brain. Is it constant throughout development or is it involved in control of development? Is the differential expressed in the developing brain?

Dr Arnaoutakis. Thank you for those questions. I will address your second point first. The candidate biomarkers that we have looked at have been developed in tandem with our collaborators in Gainesville, Florida, who specialize in proteomics, and so far as they know, developmentally, the expression of this protein is consistent.

Your first question is an especially important one because there is the issue of confounding if these biomarkers are expressed in tissues of organs that are not of interest.

UCH has 3 isoforms: L1, L2, and L3. The L1 isoform, as best we can tell, is very specific to neurons. L2 and L3 have been identified in tumors but I do not believe they are expressed in normal tissue. We do believe that UCHL1 is very specific. UCHL1 accounts for about $5 \%$ of total soluble brain protein, which may seem low, but given the gamut of the variety of brain proteins expressed, it is actually a very high amount. So we actually have seen in other models, together with Dr Wang, that this seems to be a reproducible and very specific biomarker.

Dr Michael E. Jessen (Dallas, Tex). I enjoyed your talk. This is a very important area for research. My question about this particular marker is how pragmatic it really is. It seems to me that you have to deliver a pretty severe insult to the animal before you start 
to get defining amounts of the marker in the serum and in the CSF as well. Does that detract from its clinical usefulness, whereas what we really need is something that will be detected even when subtle changes occur so that we would have the ability to intervene?

Dr Arnaoutakis. That is a great point and I appreciate your bringing that up. There are a couple of points to address in your question. The animals that we have used in our model are young, healthy, male dogs without any cerebrovascular disease or comorbidities. You are right that, to achieve damage, we have to subject these animals to a rather profound insult. However, we do believe that this has promise in humans who may have cerebrovascular disease and comorbidities and would require a less severe insult for this biomarker to be released. We think that these results support a follow-up study in humans in which we would evaluate the serum.

The other point that you bring up is especially important in the discussion of biomarkers. I know there are data both in the cardiac surgery literature and in the critical care literature regarding acute kidney injury biomarkers, for example, in which there are actually profiles of biomarkers that enhance the sensitivity. We have experience in our laboratory with biomarkers other than UCHL1. Each has a limitation. Some are not as specific to the brain as UCHL1 but are also expressed in higher levels with less severe injury.

I think ultimately what we are looking for and what we would aim to do with our human studies is not limit ourselves ahead of time by only making preparations to study a single biomarker. Rather, we would incorporate a panel so that we could increase our sensitivity and not have to rely on any single biomarker to predict devastating neurologic injury, which is frequently manifest clinically anyway.

Dr Jessen. How quickly can you get the results of an ELISA after you send the blood off so that you might have information on which you would be able to intervene?
Dr Arnaoutakis. Speaking to the logistics, at this point we are collaborating with a laboratory in a different state. Having said that, this laboratory has developed significant expertise with its ELISA and its methods. Down the road, the intention is for this to be used as a point of care test, so that it could actually be performed either in the intensive care unit or in the critical laboratory, making these results available in real time and allowing them to inform clinical decision making.

Dr Sunil P. Malhotra (New York, NY). Biomarkers of neurologic injury are a very important area for cardiac surgery in general. My question is this: You looked at 8 hours and 24 hours, but I think it is important to evaluate the reversibility of brain injury. Do you have any data on any long-term results, such as a few days out, if the markers come down and if that correlates with the canine score? Have you correlated this with any magnetic resonance imaging or axial imaging of functional assessment of brain injury?

Dr Arnaoutakis. Those are great points. The longest-term data that we have is what I presented-24 hours. It is mainly for logistical reasons through our animal care use committee and a canine model. However, we actually have preparations for a longer-term model to study these animals out to 1 week from their insult so that we can glean better information regarding the time course profile of these markers. From what we do have, it appears that the UCHL1 is actually decreasing in the serum at least at 24 hours.

From the standpoint of damage, I know that the histologic characteristics actually evolve over time such that at 8 hours, even after 2 hours of hypothermic circulatory arrest, there is not nearly as much histologic damage as what we have seen in some of our 72-hour survival animals. The other interesting point related to your question regarding longer term is that there may be biomarkers that are expressed in the period of time 48 hours to even 1 week out from injury that may shed some light on longer-term neurologic injury months down the road. That is an area that we are investigating as well. 


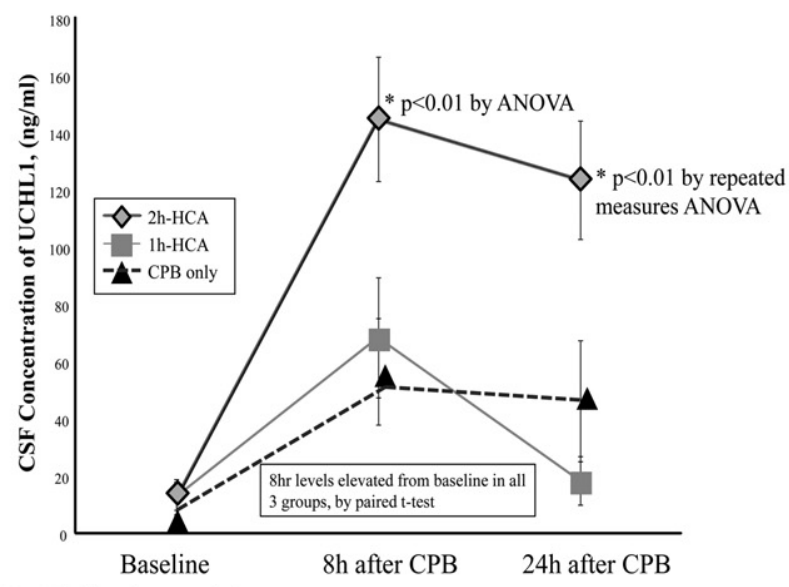

A Note: Black bars denote standard error

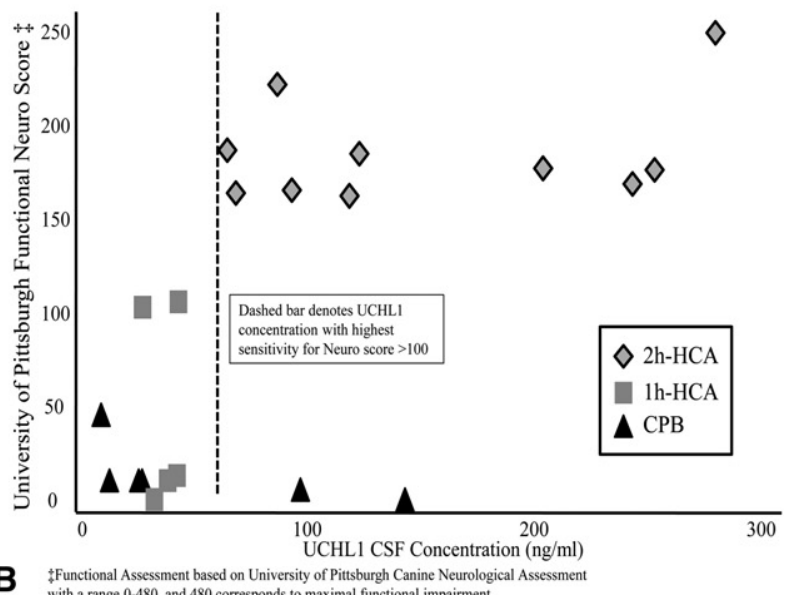
B $¥$ Functional Assessment based on University of Pittsburgh Canine Neurological Assessment

FIGURE E1. Line graph (A) depicting CSF levels of UCHL1 by treatment groups across 3 time points. Scatter plots (B) for CSF UCHL1 concentration correlated with functional assessment score. Break point denoted by dashed line indicates highest sensitivity for predicting outcome of interest (Neuro score $>100$ ) by univariate logistic regression analysis. $A N O V A$, Analysis of variance; $H C A$, hypothermic circulatory arrest; $C P B$, cardiopulmonary bypass; $U C H L 1$, ubiquitin carboxyl-terminal esterase-L1; CSF, cerebrospinal fluid.

TABLE E1. Numbers of significantly regulated genes at 8 and 24 hours after $2 \mathrm{~h}-\mathrm{HCA}, 1 \mathrm{~h}-\mathrm{HCA}$, and CPB

\begin{tabular}{|c|c|c|c|c|c|c|c|}
\hline \multirow[b]{2}{*}{ Gene } & \multicolumn{6}{|c|}{ Fold change vs normal group } & \multirow[b]{2}{*}{ Gene name } \\
\hline & $\begin{array}{l}\text { 2h-HCA } \\
\text { 8h Surv }\end{array}$ & $\begin{array}{l}\text { 2h-HCA } \\
\text { 24h Surv }\end{array}$ & $\begin{array}{l}\text { 1h-HCA } \\
\text { 8h Surv }\end{array}$ & $\begin{array}{l}\text { 1h-HCA } \\
\text { 24h Surv }\end{array}$ & $\begin{array}{c}\text { CPB } \\
\text { 8h Surv }\end{array}$ & $\begin{array}{c}\text { CPB } \\
\text { 24h Surv }\end{array}$ & \\
\hline USP21 & -1.38 & -2.05 & 1.04 & 1.14 & -1.05 & 1.06 & Ubiquitin specific peptidase 21 \\
\hline PSMB8 & 1.12 & 2.22 & -1.13 & 1.18 & 1.12 & 1.11 & $\begin{array}{l}\text { Proteasome (prosome, macropain) subunit, beta type, } 8 \\
\quad \text { (large multifunctional peptidase 7) }\end{array}$ \\
\hline PSME2 & 1.17 & 1.69 & 1.02 & 1.19 & 1.10 & -1.07 & $\begin{array}{l}\text { Proteasome (prosome, macropain) activator subunit } 2 \\
\text { (PA28 beta) }\end{array}$ \\
\hline PSMB10 & 1.16 & 1.90 & 1.03 & 1.45 & 1.31 & 1.07 & Proteasome (prosome, macropain) subunit, beta type, 10 \\
\hline PSMB9 & -1.16 & 1.58 & -1.57 & -1.19 & -1.52 & -1.16 & $\begin{array}{l}\text { Proteasome (prosome, macropain) subunit, beta type, } 9 \\
\quad \text { (large multifunctional peptidase 2) }\end{array}$ \\
\hline PSMA6 & 1.07 & 1.27 & -1.02 & 1.13 & 1.06 & -1.08 & Proteasome (prosome, macropain) subunit, alpha type, 6 \\
\hline SEPT5 & -1.24 & -1.46 & 1.21 & -1.02 & 1.04 & 1.02 & Septin 5 \\
\hline PSMB3 & 1.16 & 1.27 & 1.05 & 1.23 & 1.10 & 1.13 & Proteasome (prosome, macropain) subunit, beta type, 3 \\
\hline VHL & -1.17 & 1.04 & -1.11 & 1.01 & 1.04 & 1.01 & Von Hippel-Lindau tumor suppressor \\
\hline SNCAIP & -1.25 & -1.04 & -1.20 & -1.04 & -1.25 & -1.08 & Synuclein, alpha interacting protein \\
\hline $\mathrm{MYCN}$ & -1.25 & -1.01 & -1.41 & -1.17 & -1.36 & 1.02 & $\begin{array}{l}\text { V-myc myelocytomatosis viral related oncogene, } \\
\text { neuroblastoma derived (avian) }\end{array}$ \\
\hline
\end{tabular}

Bold numbers indicate gene terms significantly regulated for each treatment group with a false discovery rate less than 0.1. $H C A$, Hypothermic circulatory arrest; $C P B$, cardiopulmonary bypass. 